\title{
ISSUES IN MEDICINE \\ Maternal deaths associated with eclampsia in South Africa: Lessons to learn from the Confidential Enquiries into Maternal Deaths, 2005 - 2007
}

\author{
J Moodley, for the National Committee on Confidential Enquiries into Maternal Deaths, National Department of Health, South Africa
}

Hypertensive disorders of pregnancy (HDP) are the commonest medical complication in pregnancy, and remain the commonest direct cause of maternal mortality in South Africa. ${ }^{1}$ The latest $(2005$ 2007) Saving Mothers report (SMR) indicates that there were 622 maternal deaths from HDP during this period, virtually the same as in the 2002 - 2004 report. ${ }^{1,2}$ Numbers of deaths reported in the two previous SMRs were 507 for $1999-2001^{3}$ and 628 for $2002-2004 ;^{2}$ these differences probably indicate under-reporting in the triennium 1999 - 2001. The most recent reports, $2002-2004^{2}$ and $2005-2007,{ }^{1}$ better reflect the numbers of maternal deaths from HDP.

Maternal deaths in South Africa are notifiable to the Department of Health (DoH) and are reported in a structured 'Maternal death notification form', which contains demographic data and clinical details surrounding the primary and secondary causes of death. This form, together with a copy of the hospital records, is sent to the provincial Maternal, Women and Child Cluster, which has trained assessors (an experienced maternal health specialist and a midwife) to formulate a confidential opinion on any sub-standard care based on patient-related, administrative and health personnel-related factors. Assessments are collated every 3 years and published as the SMR, which includes recommendations to reduce maternal deaths.

\section{Causes of hypertensive maternal deaths in South Africa}

Table I shows the primary causes of deaths in the sub-categories of hypertensive disorders that are similar to the 2002 - 2004 report. $^{2}$ Eclampsia accounted for $55.3 \%(N=344)$ of the deaths in the period 2005 - 2007 and cerebral complications for 283 (45.5\%). Other causes of deaths included multi-organ failure, cardiac failure, metabolic complications and disseminated intravascular coagulation.

\section{Demographic data}

Of the 344 women who died from eclampsia, 171 were younger than 24 years, and of these 83 were below the age of 20 years; 50 were above the age of 35 years. Most women who died from eclampsia were also of low parity; 182 were primigravidas and 129 were of parity $1-3$.

\section{Timing of the emergency event}

A large proportion of deaths occurred in the postpartum period. Most of these women had their convulsions in the antenatal period $(N=322)$; 20 had their first convulsion in the postpartum period either in hospital, or at home after discharge from hospital.

Professor Moodley is Professor Emeritus in the Department of $\mathrm{Ob}$ stetrics and Gynaecology, University of KwaZulu-Natal. His area of research focus is women's health.
Table I. Primary obstetric causes of death in the subcategories

\begin{tabular}{lccccc}
\hline & \multicolumn{2}{c}{2005} & 2007 & & \multicolumn{2}{c}{$2002-2004$} \\
\cline { 2 - 3 } \cline { 6 - 6 } Sub-categories & $N$ & $\%$ & & $N$ & $\%$ \\
\hline Chronic hypertension & 38 & 6.1 & & 37 & 5.9 \\
Proteinuric hypertension & 173 & 27.8 & & 171 & 27.2 \\
Eclampsia & 344 & 55.3 & & 347 & 55.3 \\
HELLP syndrome & 54 & 8.7 & & 70 & 11.1 \\
Rupture of the liver & 10 & 1.6 & & 3 & 0.5 \\
Acute fatty liver & 3 & 0.48 & & 0 & 0.0 \\
$\quad$ Total & 622 & & 628 & \\
HELLP = haemolysis, elevated liver enzyme levels and a low platelet count.
\end{tabular}

\section{Avoidable factors $(N=344)$}

Patient avoidable factors were mainly related to delay in seeking help and infrequent attendance for antenatal care; $180(60 \%)$ of the 344 women who died had attended an antenatal clinic.

The major administrative factors were delay in transport between institutions (11\%) and communication problems between institutions and in institutions (11\%). Others included delay in transport from home to institution, barrier to entry, lack of accessibility, lack of health personnel, and lack of appropriately trained staff.

Health care provider avoidable factors in 336 assessable deaths related mainly to delay in referring patients $(N=63 ; 18 \%)$; management at an inappropriate level of health care facility $(N=73$; $21 \%)$; substandard care but correct diagnosis $(N=106 ; 31 \%)$; failure to diagnose $(N=68 ; 20 \%)$; and infrequent monitoring $(N=27 ; 8 \%)$.

Clinical features of severe pre-eclampsia are often not taken into account by inexperienced health personnel. In our current understanding of the causation of pre-eclampsia, delivery of the fetus and placenta leads to cure. Since these women are still at risk of complications from the disease process in the period immediately after delivery, continued frequent observations of the pulse rate, blood pressure, urinary output, level of consciousness and potential signs of pulmonary oedema must be ensured. Antihypertensive therapy must be continued and should not be stopped abruptly. Magnesium sulphate $\left(\mathrm{MgSO}_{4}\right)$ should be continued for at least 24 hours after delivery. All laboratory tests must be repeated within $4-6$ hours after delivery and results reviewed. Patients should not be discharged until their blood pressure levels have been stable for a period of at least 24 hours. Furthermore, they should be asked to return to the postnatal clinic of the hospital within a week and only referred to a community clinic when all physical and laboratory tests have returned to normal (this should have taken place within 7 days of delivery). Advice on contraception, further pregnancies and the place (level of health care facility) of future antenatal care should be provided.

\section{Discussion}

Eclampsia is the commonest direct cause of maternal death in South Africa, which is of concern since it is preventable if pre-eclampsia 
is detected early and managed by early delivery. In under-resourced countries, the availability of $\mathrm{MgSO}_{4}$ and the lack of protocols of management are significant issues associated with maternal deaths due to pre-eclampsia., ${ }^{4,5}$ In South Africa these factors should not be issues, as $\mathrm{MgSO}_{4}$ is widely available and on the essential drugs list for level 1 health facilities (district hospitals and clinics). Clinical guidelines for management of HDP, and in particular the management of obstetric emergencies, ${ }^{6,7}$ have been widely distributed to all hospitals and clinics, but are perhaps not reaching all health professionals. Regular face-to-face teaching and emergency drills on the labour ward floor may improve the situation.

Intracranial haemorrhage remains the commonest final cause of death in HDP. Although the exact mechanisms that cause hypertension and intracranial haemorrhage are not clearly understood, it appears that systolic and diastolic hypertension play a role. It is generally accepted that diastolic blood pressures of $\geq 110 \mathrm{mmHg}$ are linked with intracranial pathology, but recently systolic hypertension has also been found to play a significant role. A systolic blood pressure value above which urgent antihypertensive treatment should be given has been identified as $155-160 \mathrm{mmHg}{ }^{8}$ The latest Why Women Die publication (2003 - 2005) recommends that 'women with a systolic blood pressure of $>160 \mathrm{mmHg}$ need antihypertensive treatment. Consideration should also be given to initiating antihypertensive treatment at lower blood pressure values if the overall clinical picture suggests rapid deterioration with anticipation of severe hypertension."

In this audit, among the 207 women who had cerebral complications, the average blood pressures were systolic $177 \mathrm{mmHg}$ (range 103 - 244) and diastolic 115 (range 74 - 162). These extremely high systolic and diastolic blood pressures imply that young women with severe preeclampsia and eclampsia require rapid but smooth lowering of high blood pressure. This is particularly important as most of the patients are young with an abrupt onset of hypertension. Eighty per cent of 207 women who had convulsions were primigravidas, and their mean age was 27 years (range 13 - 45); 16 had systolic blood pressures of $>160 \mathrm{mmHg}$ with diastolic blood pressures of less than $110 \mathrm{mmHg}$. High blood pressure is of extreme importance and must be lowered rapidly, and blood pressure should be measured frequently during the acute phase. ${ }^{10}$ Although contentious, attention should also be given to the use of benzodiazepines to arrest seizures. It is likely that the blood pressures rise further during seizures and that most cerebrovascular accidents occur at this time, particularly in patients with low platelet counts. In South Africa, oral nifedipine, dihydralazine and labetalol are used for this purpose. ${ }^{7,8,11}$

In the 2005 - 2007 SMR, cardiac failure (32.8\%) and renal failure $(10.3 \%)$ were assigned as the final and contributory causes of maternal deaths (Tables I and II). ${ }^{1}$ The number of deaths due to renal failure has declined in the 2005 - 2007 report, but those from cardiac failure have increased since the 2002 - 2004 report. In the hospital records of the patients it is difficult to distinguish between death from cardiac failure and fluid overload from renal failure because cardiac failure might lead to hypotension or pulmonary oedema or both; respiratory failure also carries the inference of tachypnoea, which may develop for many reasons, including pulmonary oedema. It is likely that all these terms may indicate deaths due to pulmonary oedema. It is of concern that the number of deaths from cardiac failure (probably pulmonary oedema) have increased in the 2005 - 2007 SMR, particularly when guidelines on fluid balance are available. ${ }^{2}$ In the latest UK report (Why Women Die) there were no deaths from pulmonary causes alone, an improvement probably due to the availability of good fluid management clinical protocols. ${ }^{9}$
Table II. Final and contributory causes of maternal deaths for hypertension and a comparison with 2005 - 2007 and $2002-2004$

\begin{tabular}{lccccc}
\hline & \multicolumn{2}{c}{2005} & -2007 & & \multicolumn{2}{c}{2002} & -2004 \\
\cline { 2 - 3 } \cline { 6 - 7 } Organ system & $N$ & $\%$ & & $N$ & $\%$ \\
\hline Hypovolaemic shock & 50 & 8.0 & & 49 & 7.8 \\
Septic shock & 20 & 3.2 & & 16 & 2.5 \\
Respiratory failure & 158 & 25.4 & & 155 & 24.7 \\
Cardiac failure & 142 & 22.8 & & 89 & 14.2 \\
Renal failure & 64 & 10.3 & & 88 & 14.8 \\
Liver failure & 30 & 4.8 & & 31 & 4.9 \\
Cerebral complications & 283 & 45.5 & & 316 & 50.3 \\
Metabolic complications & 26 & 4.2 & & 7 & 1.1 \\
DIC & 70 & 11.3 & & 89 & 14.2 \\
Multi-organ failure & 88 & 14.1 & & 104 & 16.6 \\
Immune system failure & 31 & 5.0 & & 18 & 2.9 \\
Unknown & 56 & 9.0 & & 56 & 8.9 \\
DIC = disseminated intravascular coagulation. & & & \\
\hline
\end{tabular}

In a report on maternal deaths related to hypertension, one of 27 deaths between 2000 and 2004 was due to cardiac failure. The authors point out that obstetricians should be aware that postpartum mobilisation of extracellular water and subsequent shift into the intravascular compartment can aggravate hypertension. ${ }^{11}$

Fluid overload should be recognised early and treated adequately. Detecting patients at risk by history and examination, early referral and appropriate critical care is therefore essential for patients with severe pre-eclampsia.

Infrequent antenatal attendance remains a challenge. In South Africa's public hospitals most women 'book' for antenatal care, but often after the 22nd week of pregnancy, and attend infrequently. The last report emphasised community education on the benefits of antenatal care, but this appears not to have been implemented. Public health education and involvement of partners and families should again be a priority.

In the previous report ${ }^{2}$ the issue of 'hidden pregnancies' was highlighted. In this report many young women died from eclampsia; specifically, 3 teenagers aged 13, 14 and 15 years had 'hidden pregnancies', so there was considerable delay in seeking help. Education, provision of contraception and family support are key factors in preventing teenage pregnancies.

Maternity care is free of charge in South Africa and access to antenatal clinics widely available, so other social and cultural factors may be causing pregnant teenagers not to attend antenatal clinics. Improvement in education and dissemination of health information on radio, television and the print media may help to overcome this challenge. More specifically, education of antenatal attendees at time of booking and at every visit should be emphasised. Women with chronic hypertension or those with pre-eclampsia being treated as outpatients should be informed about the likely complications and steps to take if these symptoms occur.

The previous report ${ }^{2}$ also highlighted problems related to emergency management and recommendations were made regarding undergraduate, postgraduate and continuing professional education, including that all doctors should undergo a course on emergency 
resuscitation before registering as general practitioners; handson courses on mannequins done at frequent intervals are also recommended. The National Committee on Confidential Enquiries into Maternal Deaths is promoting essential steps in the management of obstetric emergencies (ESMOE). This involves trainers visiting all health districts and carrying out training on mannequins and other visual aids on the emergency management of common conditions causing maternal deaths.

Previous SMRs have not identified cases of acute fatty liver of pregnancy (AFLP), but there is an impression that this condition does occasionally occur. ${ }^{2,3}$ Whether these deaths should be grouped under HDP is debatable. AFLP is defined as a condition that occurs abruptly in the second half of pregnancy and presents with increasing jaundice and right upper quadrant pain. The liver enzymes (transaminases) are abnormal and patients go on to develop coagulopathy and encephalopathy. Treatment involves supportive measures and delivery of the fetus. On pathological examination the liver shows fatty changes in the hepatocytes. ${ }^{12}$

There were 3 cases of maternal death grouped under AFLP. All took place at the same tertiary hospital and its geographical drainage area, all the patients were relatively young ( 26 - 30 years), parity 2 and in the second half of pregnancy, and all presented with jaundice and abdominal pain. One had mild hypertension, 2 had autopsies in keeping with fatty changes in the liver, and the third was said to have had all the clinical features of AFLP. As they were all from the same geographical area, the questions of viral infections, drug-related effects, or heavy alcohol intake are raised.

\section{Conclusion}

The majority of maternal deaths from hypertensive disorders in South Africa are associated with avoidable factors and substandard care. The challenge is to implement the recommendations arising from the SMRs. There is evidence from the 2005 - 2005 report that maternal deaths due to hypertension are decreasing, but more effort needs to be put into increasing the skills of health care professionals and improving the information on maternity care that we provide to women, fathers, families and communities.

1. National Committee on Confidential Enquiries into Maternal Deaths. Saving Mothers Fourth Repor 2005-2007. Pretoria: Department of Health, 2009.

2. National Committee on Confidential Enquiries into Maternal Deaths. Saving Mothers: Third Report on Confidential Enquiries into Maternal Deaths in South Africa 2002-2004. Pretoria: Department of Health, 2005 .

3. National Committee on Confidential Enquiries into Maternal Deaths. Saving Mothers: Third Report on Confidential Enquiries into Maternal Deaths in South Africa 1999-2001. Pretoria: Department of Health, 2006.

4. The Eclampsia Trial Collaborative Group. Which anticonvulsant for women with eclampsia? Evidence from the Collaborative Eclampsia Trial. Lancet 1995;345:1455-1463.

Langer A, Villar J, Tell K, et al. Reducing eclampsia related deaths - a call to action. Lancet 2008;371:705706.

6. Department of Health. Guidelines for Maternity Care in South Africa. 3rd ed. Pretoria; Department of Health, 2007

7. Department of Health. Essential Steps in the Management of Obstetric Emergencies. Pretoria Department of Health, 2008

Martin Jr NL, Thigpen MF Moore RC, et al. Stroke and pre-eclampsia and eclampsia: a paradigm shift focussing on systolic blood pressure. Obstet Gynecol 2005;105:246-254.

9. Neilson J. Pre-eclampsia and eclampsia. In: Lewis G and CEMACH. Why Mothers Die 2003-2005. London: RCOG Press, 2007: 72

10. Paruk F, Moodley J. Untoward effects of rapid-acting antihypertensive agents. In: Arukumaran S, Moodley J, eds. Best Practice and Research Clinical Obstetrics and Gynaecology. London: Harcour Publishers, 2001: 491-506.

11. Schutte J. Schuitmaker NW, van Roosmalen J, Steeger EA. Dutch Maternal Mortality Committee. Br Obstet Gynaecol 2008:115(6):732-736.

12. Barber MA, Equiluz I, Martin A, Placencia W, Valle L, Garcia JA. Acute fatty liver of pregnancy: analysis of five consecutive cases from a tertiary centre. J Obstet Gynecol 2010; 30(3):241-243.

\section{CLINICAL IMAGES \\ Cytomegalovirus duodenitis, pseudotumour and cholangiopathy in advanced HIV}

Bradley Yudelowitz, Adam Mahomed

Gastro-intestinal cytomegalovirus (CMV) infection manifests as oesophagitis, gastritis, enteritis and colitis. ${ }^{1,2}$ Reports of duodenal involvement are rare. ${ }^{3}$ We found only one reported CMV-induced pseudotumour of the duodenum and $14 \mathrm{CMV}$-induced gastrointestinal pseudotumours. ${ }^{4-6}$

Bradley Yudelowitz was a medical officer at the Reproductive Health and HIV Research Unit, University of the Witwatersrand, Johannesburg, at the time of writing.

Adam Mahomed is clinical head of the Division of Medical Gastroenterology, Department of Internal Medicine, Charlotte Maxeke Johannesburg Academic Hospital and University of the Witwatersrand.

\section{Case report}

A 35-year-old woman presented with cholestatic jaundice and partial bowel obstruction following 3 months' history of intermittent abdominal pain, nausea and vomiting. She had been diagnosed HIV positive 2 years before presentation, and had had no further followup. An abdominal ultrasound scan demonstrated splenic microabscesses, mesenteric and para-aortic lymphadenopathy and an indiscrete mass in the region of the head of the pancreas. A computed tomography (CT) scan (Fig. 1) confirmed a mass inseparable from the first part of the duodenum (D1) in the region of the head of the pancreas, compressing the common bile duct (CBD). Magnetic resonance cholangiopancreatography (MRCP) (Fig. 2) demonstrated dilated intrahepatic ducts and a long distal CBD stricture. Empiric tuberculosis (TB) treatment was started in view of the intraabdominal lymph nodes, splenic micro-abscesses and pancreatic mass, with poor clinical response.

Because of the patient's poor clinical response to TB treatment gastroscopy was performed, and an exophytic lesion at the second part of the duodenum (D2) was encountered which the scope was 